ALEA, Lat. Am. J. Probab. Math. Stat. 17, 355-374 (2020)

DOI: $10.30757 /$ ALEA.v17-14

\title{
Some remarks on associated random fields, random measures and point processes
}

\section{Günter Last, Ryszard Szekli and Dhandapani Yogeshwaran}

Karlsruhe Institute of Technology

Institute for Stochastics,

76131 Karlsruhe, Germany.

E-mail address: guenter.last@kit.edu

URL: http://www.math.kit.edu/stoch/ last/

University of Wrocław,

Mathematical Institute,

50-384, Wrocław, Poland.

E-mail address: szekli@math.uni.wroc.pl

URL: http://www.math.uni.wroc.pl/ szekli/

Theoretical Statistics and Mathematics Unit, Indian Statistical Institute,

Bangalore, India

E-mail address: d.yogesh@isibang.ac.in

$U R L:$ https://www.isical.ac.in/yogeshwaran-dhandapani

\begin{abstract}
In this paper, we first show that for a countable family of random elements taking values in a partially ordered Polish space with a closed partial order (POP space), association (both positive and negative) of all finite dimensional marginals implies that of the infinite sequence. Our proof proceeds via Strassen's theorem for stochastic domination and thus avoids the assumption of normally ordered on the product space as needed for positive association in Lindqvist (1988). We use these results to show on POP spaces that finite dimensional negative association implies negative association of the random measure and negative association is preserved under weak convergence of random measures. The former provides a simpler proof in the most general setting of Polish spaces complementing the recent proofs in Poinas et al. (2019) and Lyons (2014) which restrict to point processes in $\mathbb{R}^{d}$ and locally compact Polish spaces respectively. We also provide some examples of associated random measures which shall illustrate our results as well.
\end{abstract}

Received by the editors March 14th, 2019; accepted March 13th, 2020.

2010 Mathematics Subject Classification. 60E15, 60G57.

Key words and phrases. negative association, positive association, random fields, random measures, point processes, weak convergence, Gaussian random fields, Poisson processes, Cox processes, Poisson cluster processes determinantal point processes, Gibbs point processes.

Research supported by: first author, German Research Foundation through Grant No. LA965/9-2 awarded as part of the DFG-Forschungsgruppe FOR 1548; second author, National Science Centre, Poland, grant NCN no 2015/19/B/ST1/01152; third author, INSPIRE Faculty Award from DST and CPDA grant from the Indian Statistical Institute. 


\section{Introduction}

Positive association of random vectors in $\mathbb{R}^{d}$ appears in Esary et al. (1967), and negative association several years later, see Joag-Dev and Proschan (1983) or Alam and Saxena (1981). Since then the theory of positive association has been well developed and has found many applications in various contexts, for example to establish limit theorems, to obtain concentration bounds or to derive stochastic comparison results. Association of real random fields on $\mathbb{Z}^{d}$ and $\mathbb{R}^{d}$ were used to obtain central limit theorems for random fields, see e.g. Bulinski and Shashkin (2007), Bulinski and Spodarev (2013) or Poinas et al. (2019) and references therein. We shall give examples of associated (positive and negative) random fields later in Section 5 and in the Appendix. Positive association of probability measures on partially ordered Polish spaces was studied in Lindqvist (1988), where infinite products of such spaces and some space of functions with values in partially ordered Polish spaces were characterized by the corresponding finite dimensional distributions under the additional assumption that the product space is normally ordered. Inspired by one of the proofs in Georgii and Yoo (2005), we use Strassen's theorem on stochastic domination to prove the characterization by finite dimensional distributions for both positive and negative association for countable families of random elements of general partially ordered Polish spaces (see Theorem 3.3), which generalizes Theorem 5.1 in Lindqvist (1988). Using this idea, we characterize negative association by bounded, continuous, non-decreasing functions (Lemma 3.5) and also show that association for countable families of random elements in partially ordered Polish spaces is preserved under weak convergence (Theorem 3.6).

A special case of partially ordered Polish spaces is that of the space of all locally finite measures and in particular the space of locally finite counting measures. These two spaces are of importance in the theory of random measures and point processes. Positive association of random measures and point processes on locally compact Polish spaces were characterized by the corresponding finite dimensional distributions by Kwieciński and Szekli (1996, Theorem 3.2). Using our Theorem 3.3, we will prove an analogous characterization (by finite dimensional distributions) of negative association for random measures on Polish spaces (Theorem 4.4). Similar results on negative association for point processes on $\mathbb{R}^{d}$ and on locally compact Polish spaces has been recently given by Poinas et al. (2019, Theorem 2.3) and Lyons (2014, paragraph 3.7) respectively. Though the latter result is in the context of determinantal point processes, the proof applies to general negatively associated point processes. We will compare these theorems in more detail in Section 4. We will extend these results into the context of random measures and will also relax a rather restrictive assumption of local compactness on the ground space. We use this along with Theorem 3.6 to show that weak convergence of random measures also preserves negative association (Theorem 4.6). Apart from giving a very general characterization of negatively associated random elements, our result opens new possibilities, for example to obtain central limit theorems for associated random measures in a quite general context. Our results allow to extend several association properties of countable random fields known only for finite dimensional distributions into the setting of infinite sequences as well as generate new examples of negatively associated random measures (see Section 5).

We end the introduction with a brief discussion of the theory of negative dependence. Though negative association was introduced in Joag-Dev and Proschan 
(1983) and Alam and Saxena (1981) in the context of reliability models, it garnered significant additional interest following the article of Pemantle (2000) in which he confined himself to binary-valued random variables. The list of examples that motivated him to develop techniques for proving that measures have negative dependence properties such as negative association or strong Rayleigh property include uniform random spanning trees, simple exclusion processes, random cluster models and the occupation status of competing urns. Among various definitions expressing negative dependence, negative association seems to be one of the easier conditions to verify and has also found applications. Negative association has one distinct advantage over the other types of negative dependence, namely, nondecreasing functions of disjoint sets of negatively associated random variables are also negatively associated. This closure property does not hold for the other types of negative dependence. There exists nothing like a general theory of negative association on partially ordered Polish spaces, no reasonable analogy to the theory of positive association is visible. New examples of negatively associated point processes and random measures are given in Last and Szekli (2019) along with some stochastic comparisons of dependence. Positive association properties proved for many interacting particle systems stay in contrast with the lack of negative association results for most interacting particle systems. A property related to negative association, known to hold for symmetric exclusion processes is the strong Rayleigh property (stronger than negative association) which is preserved in the evolution of the symmetric exclusion process (see Borcea et al., 2009, Theorem 5.2). The article is organized as follows. We introduce partially ordered Polish spaces and Strassen's theorem in Section 2 and then present our results about countable family of associated random elements in Section 3. We then state and prove our results on associated random measures in Section 4 and conclude with various (old and new) examples of associated random elements in Section 5. At the end of this paper, in an appendix, we present some additional examples which are directly related to some applied stochastic models in order to gain a broader view over this field. All formulations in the listed examples are in a strong sense PA and NA as given in Definitions 3.1 and 4.2. In many cases the exisiting results are known only for finite dimensional vectors but we extend this to the infinite-dimensional vectors using our results.

\section{Preliminaries}

Let $\mathbb{X}$ be a Polish space endowed with a partial ordering $\preceq$. A real-valued function $f$ on $\mathbb{X}$ is said to be non-decreasing if $x \preceq y$ implies $f(x) \leq f(y)$. We let $\mathcal{X}$ denote the Borel $\sigma$-field on $\mathbb{X}$. For probability measures $P$ and $P^{\prime}$ on $(\mathbb{X}, \mathcal{X}), P$ is stochastically dominated by $P^{\prime}$ if

$$
\int f d P \leq \int f d P^{\prime}
$$

for all non-decreasing bounded measurable $f$. In this case we write $P \preceq_{s t} P^{\prime}$. We assume that the partial ordering $\preceq$ is closed, i.e., the set $H=\left\{\left(x, x^{\prime}\right) ; x \preceq x^{\prime}\right\}$ is closed in the product topology on $\mathbb{X}^{2}$. For the reader's convenience we state the classical (Strassen) theorem on stochastic domination:

Theorem 2.1. $P$ and $P^{\prime}$ satisfy $P \preceq$ st $P^{\prime}$ iff there exists a probability measure $\tilde{P}$ on $\left(\mathbb{X}^{2}, \mathcal{X}^{2}\right)$ with marginals $P$ and $P^{\prime}$ such that $\tilde{P}(H)=1$. 
This result is often referred to as "Strassen's Theorem", which is formally misleading: in Strassen (1965) it is only briefly mentioned as one possible application of Theorem 11 in that paper, and the condition $P \preceq_{s t} P^{\prime}$ does not appear explicitly. An explicit formulation can be found in Kamae et al. (1977, Theorem 1). For a nice proof of Theorem 2.1 and some additional observations, see Lindvall (1999). It is known (see Kamae et al., 1977, Theorem 2) that the relation $\preceq_{s t}$ on the space of probability measures on $(\mathbb{X}, \mathcal{X})$ with the topology of weak convergence is a closed partial ordering.

\section{Association of discrete random fields}

Let $I$ be countable index set (e.g. $I=\{1, \ldots, n\}, I=\mathbb{Z}^{d}, I=\mathbb{Z}_{+}$). Let $\mathbf{X}=\left(X_{i}\right)_{i \in I}$ be a random field, that is a family of random elements with values in a partially ordered Polish (POP) space $(\mathbb{X}, \mathcal{X})$. For $J \subset I$, we write $X_{J}:=\left(X_{i}\right)_{i \in J}$.

Definition 3.1. For a family $\mathbf{X}=\left(X_{i}\right)_{i \in I}$ of random elements of $(\mathbb{X}, \mathcal{X})$

(i) we say that $\mathbf{X}$ is negatively associated (NA) if

$$
\mathbb{E}\left[f\left(X_{J}\right) g\left(X_{J^{c}}\right)\right] \leq \mathbb{E}\left[f\left(X_{J}\right)\right] \mathbb{E}\left[g\left(X_{J^{c}}\right)\right]
$$

for all $J \subset I$ and for all (coordinatewise) non-decreasing bounded measurable $f: \mathbb{X}^{J} \rightarrow \mathbb{R}$ and $g: \mathbb{X}^{J^{c}} \rightarrow \mathbb{R}$, where $\mathbb{X}^{J}$ denotes the space of all $\mathbb{X}$ valued functions defined on $J$;

(ii) we say that $\mathbf{X}$ is positively associated (PA) if

$$
\mathbb{E}\left[f\left(X_{J}\right) g\left(X_{J}\right)\right] \geq \mathbb{E}\left[f\left(X_{J}\right)\right] \mathbb{E}\left[g\left(X_{J}\right)\right]
$$

for all $J \subset I$ and for all (coordinatewise) non-decreasing bounded measurable $f: \mathbb{X}^{J} \rightarrow \mathbb{R}$ and $g: \mathbb{X}^{J} \rightarrow \mathbb{R}$, where $\mathbb{X}^{J}$ denotes the space of all $\mathbb{X}$ valued functions defined on $J$.

Remark 3.2. Association for uncountable index sets $I$ can be defined as follows. For a family $\mathbf{X}=\left(X_{i}\right)_{i \in I}$ of random elements of $(\mathbb{X}, \mathcal{X})$ we say that $\mathbf{X}$ is positively associated (PA) if

$$
\mathbb{E}\left[f\left(X_{J}\right) g\left(X_{J}\right)\right] \geq \mathbb{E}\left[f\left(X_{J}\right)\right] \mathbb{E}\left[g\left(X_{J}\right)\right]
$$

for all countable $J \subset I$ and for all (coordinatewise) non-decreasing bounded measurable $f: \mathbb{X}^{J} \rightarrow \mathbb{R}$ and $g: \mathbb{X}^{J} \rightarrow \mathbb{R}$. Similarly, one can define NA using disjoint countable index sets $J, J^{\prime} \subset I$.

By the well-known formula

$$
\mathbb{C o v}[X, Y]=\int \operatorname{Cov}[\mathbf{1}\{X>s\}, \mathbf{1}\{Y>t\}] d(s, t),
$$

valid for all integrable real random variables $X$ and $Y$ with $\mathbb{E}[|X Y|]<\infty$, it is enough to assume in (3.1) that $f$ and $g$ are non-negative. The above identity can be found in the proof of Lehmann (1966, Lemma 2) which the author attributes to Höffding (1940).

We say that a family $\mathbf{X}=\left(X_{i}\right)_{i \in I}$ of random elements of $(\mathbb{X}, \mathcal{X})$ is associated if it is PA or NA. In our proofs we will concentrate on the NA case, only pointing out how to deal with the NA case. 
We now state and prove one of our main theorems showing that NA property of finite dimensional marginals implies that of the infinite sequence. Our proof was inspired by the proof of Georgii and Yoo (2005, Corollary 3.4).

Theorem 3.3. Consider a discrete family $\mathbf{X}=\left(X_{n}\right)_{n \in I}$ of random elements of POP space $(\mathbb{X}, \mathcal{X})$. Assume that for each finite $J \subset I$ the finite subfamily $X_{J}$ is associated. Then the family $\mathbf{X}$ is associated in the same positive or negative way as finite subfamilies.

Proof: We prove the NA case. In order to check (3.1), let us first assume that $J \subset I$ is finite and because of $(3.4)$, let $f: \mathbb{X}^{J} \rightarrow[0, \infty)$ be non-decreasing and such that $\mathbb{E}\left[f\left(X_{J}\right)\right]<\infty$. It is no restriction of generality to assume that $\mathbb{E}\left[f\left(X_{J}\right)\right]>0$. (Otherwise we have that $\mathbb{P}\left(f\left(X_{J}\right)=0\right)=1$ and (3.1) becomes trivial.) Since we assumed that $I$ is discrete, we can enumerate elements of $I$ and assume that $J=\{1, \ldots, m\}$ for some $m \in \mathbb{Z}_{+}$. For $n \in \mathbb{Z}_{+}$, we define a random element $X_{J}^{(n)}=\left(X_{k}^{(n)}\right)_{k \geq 1}$ of $\mathbb{X}^{\mathbb{Z}_{+}}$by $X_{k}^{(n)}:=X_{m+k}$ for $k \in\{1, \ldots, n\}$ and $X_{k}^{n}:=z$ for $k \notin$ $\{1, \ldots, n\}$, for a fixed element $z \in \mathbb{X}$. We define a random element $X_{J}^{\infty}=\left(X_{k}^{\infty}\right)_{k \geq 1}$ of $\mathbb{X}^{\mathbb{Z}_{+}}$by $X_{k}^{\infty}:=X_{m+k}$ for $k \geq 1$. By our assumption, we have that for all $n \in \mathbb{Z}_{+}$

$$
\mathbb{E}\left[f\left(X_{J}\right) g\left(X_{J}^{(n)}\right)\right] \leq \mathbb{E}\left[f\left(X_{J}\right)\right] \mathbb{E}\left[g\left(X_{J}^{(n)}\right)\right]
$$

for all measurable non-decreasing $f, g: \mathbb{X}^{\mathbb{Z}_{+}} \rightarrow \mathbb{R}$ such that $\mathbb{E}\left[\left|g\left(X_{J}^{(n)}\right)\right|\right]<\infty$. But this means that

$$
\mu_{n, J} \preceq_{s t} \nu_{n, J}, \quad n \in \mathbb{Z}_{+},
$$

where $\mu_{n, J}:=\mathbb{E}\left[f\left(X_{J}\right)\right]^{-1} \mathbb{E}\left[f\left(X_{J}\right) \mathbf{1}\left\{X_{J}^{(n)} \in \cdot\right\}\right], \nu_{n, J}:=\mathbb{P}\left(X_{J}^{(n)} \in \cdot\right)$ and $\preceq_{s t}$ denotes strong stochastic ordering of probability measures on $\mathbb{X}^{\mathbb{Z}_{+}}$(w.r.t. coordinatewise $\preceq$ ordering). Moreover, the set

$$
H:=\left\{(x, y) \in \mathbb{X}^{\mathbb{Z}_{+}} \times \mathbb{X}^{\mathbb{Z}_{+}}: x \preceq y\right\}
$$

is closed w.r.t. the product topology on $\mathbb{X}^{\mathbb{Z}_{+}} \times \mathbb{X}^{\mathbb{Z}_{+}}$. By Strassen's theorem there exists for each $n \in \mathbb{Z}_{+}$a probability measure $\gamma_{n, J}$ on $\mathbb{X}^{\mathbb{Z}_{+}} \times \mathbb{X}^{\mathbb{Z}_{+}}$with marginals $\mu_{n, J}$ and $\nu_{n, J}$, respectively, such that $\gamma_{n, J}(H)=1$.

By Kallenberg (2002, Theorem 4.29) we have that $\mu_{n, J} \stackrel{d}{\rightarrow} \mu_{J}$ as $n \rightarrow \infty$, where

$$
\mu_{J}:=\mathbb{E}\left[f\left(X_{J}\right)\right]^{-1} \mathbb{E}\left[f\left(X_{J}\right) \mathbf{1}\left\{X_{J}^{\infty} \in \cdot\right\}\right]
$$

Similarly, $\nu_{n, J} \stackrel{d}{\rightarrow} \nu_{J}:=\mathbb{P}\left(X_{J}^{\infty} \in \cdot\right)$.

Now we use a similar argument as in Kamae et al. (1977, Proposition 3). By Kallenberg (2002, Theorem 16.3) we have that the sequences $\left(\mu_{n, J}\right)$ and $\left(\nu_{n, J}\right)$ are tight. Since $\gamma_{n, J}$ has marginals $\mu_{n, J}$ and $\nu_{n, J}$ we have for any measurable $A, B \subset \mathbb{X}^{\mathbb{Z}_{+}}$that

$$
\begin{aligned}
\gamma_{n, J}\left((A \times B)^{c}\right) & =\gamma_{n, J}\left(A^{c} \times B\right)+\gamma_{n, J}\left(A \times B^{c}\right)+\gamma_{n, J}\left(A^{c} \times B^{c}\right) \\
& \leq 2 \mu_{n, J}\left(A^{c}\right)+\nu_{n, J}\left(B^{c}\right) .
\end{aligned}
$$

Therefore the sequence $\left(\gamma_{n, J}\right)$ is also tight. Let $\gamma_{J}$ be a subsequential limit. Since $H$ is closed, the Portmanteau theorem shows that $\gamma_{J}(H)=1$. By definition of weak convergence, $\gamma_{J}$ has marginals $\mu_{J}$ and $\nu_{J}$, respectively. But this implies that $\int g d \mu_{J} \leq \int g d \nu_{J}$ for all measurable non-decreasing bounded $g: \mathbb{X}^{\mathbb{Z}_{+}} \rightarrow[0, \infty)$, so that (3.1) follows. 
Finally we take an arbitrary $J \subset \mathbb{Z}_{+}$. By the first step of the proof we have

$$
\mathbb{E}\left[f\left(X_{J}\right) g\left(X_{J^{\prime}}\right)\right] \leq \mathbb{E}\left[f\left(X_{J}\right)\right] \mathbb{E}\left[g\left(X_{J^{\prime}}\right)\right]
$$

for all finite sets $J^{\prime} \subset \mathbb{Z}_{+} \backslash J$, for all non-decreasing measurable bounded $f: \mathbb{X}^{J} \rightarrow \mathbb{R}$ and $g: \mathbb{X}^{J^{\prime}} \rightarrow \mathbb{R}$. Repeating the above arguments yields (3.1) in full generality.

The proof for the PA case can be done in a similar way.

Our proof technique gives the following corollary.

Corollary 3.4. Suppose $\mathbf{X}=\left(X_{n}\right)_{n \in I}$ and $\mathbf{Y}=\left(Y_{n}\right)_{n \in J}$ are two discrete families of random elements of POP space $(\mathbb{X}, \mathcal{X})$. Assume that for all finite $I^{\prime}, J^{\prime}$ and non-decreasing bounded measurable $f, g$, it holds that

$$
\mathbb{E}\left[f\left(X_{I^{\prime}}\right) g\left(Y_{J^{\prime}}\right)\right] \leq(\geq) \mathbb{E}\left[f\left(X_{I^{\prime}}\right)\right] \mathbb{E}\left[g\left(Y_{J^{\prime}}\right)\right] .
$$

Then, for all non-decreasing bounded measurable $f, g$ and countable $I, J$, we have that

$$
\mathbb{E}\left[f\left(X_{I}\right) g\left(Y_{J}\right)\right] \leq(\geq) \mathbb{E}\left[f\left(X_{I}\right)\right] \mathbb{E}\left[g\left(Y_{J}\right)\right]
$$

A second very useful consequence of our proof technique is the following lemma allowing us to restrict (3.1) to only bounded continuous non-decreasing functions.

Lemma 3.5. Consider a finite family $\mathbf{X}=\left(X_{1}, \ldots, X_{m}\right)$ such that it satisfies (3.1) ((3.2)) for all non-negative, bounded, continuous, non-decreasing functions $f, g$ on $\mathbb{X}^{J}, \mathbb{X}^{K}$ respectively where $J \subset\{1, \ldots, m\}$ and $K=\{1, \ldots, m\} \backslash J$. Then $\mathbf{X}$ is NA (PA).

Proof: We shall again prove in the case of NA alone. Let $J, K$ be as assumed in the lemma. From (3.4), it suffices to show (3.1) for all non-negative bounded, measurable non-decreasing functions. Let $g$ be a non-negative, bounded, continuous, non-decreasing function such that $\mathbb{E}\left[g\left(X_{K}\right)\right]>0$. Thus, we have for all non-negative, bounded, continuous, non-decreasing functions $f$ that

$$
\mathbb{E}\left[f\left(X_{J}\right) g\left(X_{K}\right)\right] \leq \mathbb{E}\left[f\left(X_{J}\right)\right] \mathbb{E}\left[g\left(X_{K}\right)\right],
$$

and this inequality can be re-written in the form of a stochastic order relation as

$$
\mathbb{E}\left[g\left(X_{K}\right]^{-1} \mathbb{E}\left[f\left(X_{J}\right) g\left(X_{K}\right)\right] \leq \mathbb{E}\left[f\left(X_{J}\right)\right] .\right.
$$

Defining probability measures $\nu_{J}:=\mathbb{P}\left(X_{J} \in \cdot\right), \mu_{J}^{g}:=\mathbb{E}\left[g\left(X_{K}\right)\right]^{-1} \mathbb{E}\left[g\left(X_{K}\right) \mathbf{1}\left\{X_{J} \in\right.\right.$ $.$\} ], we have that the above inequality implies \mu_{J}^{g} \preceq_{s t} \nu_{J}$ by Müller and Stoyan (2002, Theorem 2.6.4). From the definition of $\preceq_{s t}$ (i.e., stochastic domination), we have that

$$
\mathbb{E}\left[f\left(X_{J}\right) g\left(X_{K}\right)\right] \leq \mathbb{E}\left[f\left(X_{J}\right)\right] \mathbb{E}\left[g\left(X_{K}\right)\right],
$$

for all non-negative, bounded, measurable functions $f$. Now repeating the above argument by fixing a non-negative bounded measurable function $f$ such that $\mathbb{E}\left[f\left(X_{J}\right)\right]>0$, we can derive that (3.1) holds for all non-negative, bounded, measurable non-decreasing functions $f, g$ as required to complete the proof.

A powerful consequence of the above lemma is that the property of association is preserved under weak convergence. We shall use this theorem in our next section on random measures but only in the case of $\mathbb{X}=\mathbb{R}$. 
Theorem 3.6. For $k \geq 1$, consider a discrete family $\mathbf{X}^{k}=\left(X_{i}^{k}\right)_{i \in I}$ of random elements of POP space $(\mathbb{X}, \mathcal{X})$. Assume that $\mathbf{X}^{k}$ is associated for every $k \geq 1$ in the same way (i.e., always $\mathrm{PA}$ or always $\mathrm{NA}$ ) and $\mathbf{X}^{k} \stackrel{d}{\rightarrow} \mathbf{X}$ as $k \rightarrow \infty$. Then, $\mathbf{X}$ is associated in the same positive or negative way as the elements in the sequence.

Proof: From our assumptions, we have that for each $k \geq 1, m \geq 1,\left(X_{1}^{k}, \ldots, X_{m}^{k}\right)$ is associated, and $\left(X_{1}^{k}, \ldots, X_{m}^{k}\right) \stackrel{d}{\rightarrow}\left(X_{1}, \ldots, X_{m}\right)$. Thus, we have that $\left(X_{1}, \ldots, X_{m}\right)$ satisfies (3.1) (or (3.2)) for for all non-negative, bounded, continuous, nondecreasing functions $f, g$ defined on disjoint index sets of $\{1, \ldots, m\}$. Now, from Lemma 3.5 we have that $\left(X_{1}, \ldots, X_{m}\right)$ is a finite NA (or PA) family and because of our Theorem 3.3, this suffices to conclude that $\mathbf{X}$ is a NA (or PA) family.

We now compare our above results and proof techniques to those in the literature. Under the assumption that the product POP space is normally ordered, Lemma 3.5 and Theorem 3.6 are shown for PA in Lindqvist (1988, Theorem 3.1(v)). Lemma 3.5 for PA is shown for $\mathbb{X}=\mathbb{R}$ in Esary et al. (1967, Lemma 3.1 and Theorem 3.3). The proof techniques of Esary et al. (1967) and Lindqvist (1988) involve approximating binary, non-decreasing, measurable functions by non-negative, bounded, continuous, non-decreasing functions and these require additional assumptions on the space $\mathbb{X}$ relating the metric and order. These ideas can also be implemented in the case of NA with suitable modifications. However, our proof avoids these by using Strassen's theorem and similar criteria holding for stochastic domination.

An alternative assumption to normally ordered spaces is the following condition formulated in Rüschendorf (1981) (recalled as (R1) in Lindqvist, 1988): $x \mapsto d(x, A)$ is non-increasing for an increasing set $A$. Under this assumption, the proof ideas as in Esary et al. (1967, Lemma 3.1 and Theorem 3.3) or Lindqvist (1988, Theorem 3.1(v)) or Rüschendorf (1981, Theorem 1(d)) can be adapted suitably for both $\mathrm{PA}$ and NA. Also, we would like to mention that this condition and the property of being normally ordered need not be related (see Noebels, 1981, pg. 38).

\section{Association of random measures}

Let $\mathbb{S}$ be a Polish space, $\mathcal{S}$ be the $\sigma$-field of Borel subsets of $\mathbb{S}$, and $\mathcal{S}_{b}$ be the ring of bounded Bore1 sets in $\mathbb{S}$. By a random measure $M$ on $\mathbb{S}$ we mean a mapping of some probability space $(\Omega, \mathcal{F}, P)$ into the space $\mathbf{M}(\mathbb{S})$ of Radon measures on $(\mathbb{S}, \mathcal{S})$, equipped with the smallest $\sigma$-field making the mappings $\mu \mapsto \mu(B)$ measurable for all $B \in \mathcal{S}$. When $M$ is a.s. confined to the space $\mathbf{N}(\mathbb{S}) \subset \mathbf{M}(\mathbb{S})$ of integer valued measures, we say that $M$ is a point process. Vague convergence $\mu_{n} \rightarrow \mu$ in $\mathbf{M}(\mathbb{S})$ means that

$$
\int_{\mathbb{S}} f d \mu_{n} \rightarrow \int_{\mathbb{S}} f d \mu
$$

for each continuous $f: \mathbb{S} \rightarrow \mathbb{R}_{+}$with bounded support. A natural partial ordering on $\mathbf{M}(\mathbb{S})$ and $\mathbf{N}(\mathbb{S})$ is given by: $\mu<\nu$ if $\mu(B) \leq \nu(B)$, for all $B \in \mathcal{S}_{b}$. It is known Rolski and Szekli (1991, Lemma 1) that the vague topology and the partial order $<$ are related, namely $<$ is closed, i.e. the set $\{(\mu, \nu): \mu<\nu\} \subset \mathbf{M}(\mathbb{S})^{2}$ is closed in the product topology on $\mathbb{S}^{2}$.

We denote the strong stochastic ordering of random elements of $\mathbf{M}(\mathbb{S})$ by $<_{s t}$. A random measure $M$ is then said to be positively associated (PA)

$$
\mathbb{E}[f(M) g(M)] \geq \mathbb{E}[f(M)] \mathbb{E}[g(M)]
$$


for any pair of real valued, bounded measurable functions $f, g$ on $\mathbf{M}(\mathbb{S})$, nondecreasing w.r.t. the order $<$.

Let $\mathcal{I} \subset \mathcal{S}_{b}$ be a countable, topological, dissecting, semi-ring generating the $\sigma$ field $\mathcal{S}$, as defined in Kallenberg (2017, Lemma 1.9). Denote by $I_{1}, I_{2}, \ldots$ some enumeration of the elements of $\mathcal{I}$. Define the mapping $\gamma: \mathbf{M}(\mathbb{S}) \rightarrow \mathbb{R}_{+}^{\infty}$ by

$$
\gamma(\mu):=\left(\mu\left(I_{1}\right), \mu\left(I_{2}\right), \ldots\right)
$$

and let $\mathbb{G}:=\gamma(\mathbb{S})$. Since $\mathcal{I}$ is a semi-ring generating $\mathcal{S}$, by Billingsley (1995, Theorem 11.3) the mapping $\gamma$ is $1-1$ and it is also increasing. Let $\rho$ be a complete metric in $\mathbb{S}$ generating the vague topology. Define a metric $\rho_{\gamma}$ in $\mathbb{G}$ by

$$
\rho_{\gamma}(x, y)=\rho\left(\gamma^{-1}(x), \gamma^{-1}(y)\right),
$$

for all $x, y \in \mathbb{G}$. We recall some basic properties of $\mathbb{G}$; see Rolski and Szekli (1991, Lemma 2) and Kallenberg (2002, Theorem A1.3).

\section{Lemma 4.1.}

(i) We have that $\mathbb{G} \in \mathcal{B}\left(\mathbb{R}_{+}^{\infty}\right)$ and that the inverse map $\gamma^{-1}: \mathbb{G} \rightarrow \mathbf{M}(\mathbb{S})$ is measurable.

(ii) $\mathbb{G}$ is metrizable as a Polish space by the metric $\rho_{\gamma}$.

(iii) The Borel $\sigma$-field $\mathcal{B}(\mathbb{G})$ generated by $\rho_{\gamma}$ is of the form $\mathcal{B}(\mathbb{G})=\mathbb{G} \cap \mathcal{B}\left(\mathbb{R}_{+}^{\infty}\right)$.

For a Borel set $A \subset \mathbb{S}$, let $\mathcal{F}(A)$ denote the $\sigma$-field on $\mathbf{N}(\mathbb{S})$ generated by the functions $\mu \mapsto \mu(B)$ for Borel $B \subseteq A$. We say that a function on $\mathbf{N}(\mathbb{S})$ is measurable with respect to $A$ if it is measurable with respect to $\mathcal{F}(A)$. For each measure $\mu$ on $\mathbb{S}$, we denote by $\mu_{A}:=\mu(\cdot \cap A)$ the restriction of $\mu$ to $A$. Then a measurable function $f: \mathbf{M}(\mathbb{S}) \rightarrow \mathbb{R}$ is $A$-measurable iff $f(\mu)=f\left(\mu_{A}\right)$ for each $\mu \in \mathbf{M}(\mathbb{S})$.

The following definition is an extension to random measures of definitions used by Lyons (2014) and Poinas et al. (2019) for point processes.

Definition 4.2. We say that a random measure $M$ is negatively associated (NA) if

$$
\mathbb{E}[f(M) g(M)] \leq \mathbb{E}[f(M)] \mathbb{E}[g(M)],
$$

for every pair $f, g$ of bounded non-decreasing functions that are measurable with respect to disjoint measurable subsets of $\mathbb{S}$.

Remark 4.3. The above definition of NA property for random measures is not equivalent to the one given in Remark 3.2 when random measures are viewed as random fields indexed by the uncountable set $\{B: B \in \mathcal{S}\}$. But for PA property, these two definitions - (4.1) and that in Remark 3.2 - are equivalent.

We shall again refer to a random measure as associated if it is either negatively associated or positively associated. As a consequence of Lemma 4.1, Kwieciński and Szekli (1996, Theorem 3.2) proved for locally compact spaces that the random measure $M$ is positively associated iff random vectors $\left(M\left(B_{1}\right), \ldots M\left(B_{n}\right)\right)$ are positively associated for all $n \geq 1$, and bounded sets $B_{1}, \ldots, B_{n} \in \mathcal{S}_{b}$. We next show an analogous result for the NA-property. We shall relax the assumption on local compactness. To get the positive association result it was enough to use the fact that non-decreasing transformations of positively associated random elements into another partially ordered space are again positively associated elements of this space. For negative association this property does not hold. In Poinas et al. (2019, Theorem 2.3) the proof of an analog of Theorem 4.4 is given for point processes 
on $\mathbb{S}=\mathbb{R}^{d}$. They use a variant of the monotone class theorem. This proof is not directly applicable for more general spaces $\mathbb{S}$. A proof of the NA part of Theorem 4.4 for point processes on locally compact partially ordered Polish spaces can be (implicitly) found in Lyons (2014, paragraph 3.7), where negative association of some determinantal point processes on locally compact Polish spaces is proved. The arguments there are rather lengthy and are based on Lusin's separation theorem and the Choquet capacitability theorem. We shall give a short proof of this result in a more general setting of random measures, using Lemma 4.1 and Theorem 3.3.

Theorem 4.4. Let $M$ be a random measure on a Polish space $\mathbb{S}$. Then $M$ is associated if and only if random vectors $\left(M\left(B_{1}\right), \ldots M\left(B_{n}\right)\right)$ are associated in the same positive or negative way for all $n \geq 1$, and disjoint sets $B_{1}, \ldots, B_{n} \in \mathcal{I}$.

Before proving the theorem, we need a lemma that will allow us to assume that the bounded disjoint sets can be taken to be measurable in the above theorem instead of just elements of $\mathcal{I}$.

Lemma 4.5. Let $M$ be a random measure on a Polish space $\mathbb{S}$.

Then $\left(M\left(B_{1}\right), \ldots, M\left(B_{n}\right)\right)$ is associated for all $n \geq 1$, and disjoint sets $B_{1}, \ldots, B_{n} \in \mathcal{I}$ iff $\left(M\left(B_{1}\right), \ldots, M\left(B_{n}\right)\right)$ is associated in the same positive or negative way for all $n \geq 1$ and disjoint sets $B_{1}, \ldots, B_{n} \in \mathcal{S}_{b}$.

Proof: We shall again prove only for NA property and the same proof applies more easily to PA. The 'if' part is trivial as $\mathcal{I} \subset \mathcal{S}_{b}$ and we shall now prove the other part. Fix $m$ and disjoint $B_{2}, \ldots, B_{m} \in \mathcal{I}$ and consider the class $\mathcal{M}$ of all bounded measurable sets $B$ such that $\left(M\left(B \backslash\left(B_{2} \cup \ldots \cup B_{m}\right)\right), M\left(B_{2}\right), \ldots, M\left(B_{m}\right)\right)$ is NA. If $B \in \mathcal{I}$, then $B \backslash\left(B_{2} \cup \ldots \cup B_{m}\right)$ can be written as a finite disjoint union of $\mathcal{I}$-sets and hence $\left(M\left(B \backslash\left(B_{2} \cup \ldots \cup B_{m}\right)\right), M\left(B_{2}\right), \ldots, M\left(B_{m}\right)\right)$ is NA. So, $\mathcal{I} \subset \mathcal{M}$. Denoting by $\mathcal{R}(\mathcal{I})$, the ring generated by taking finite unions of sets in $\mathcal{I}$, we have that $\mathcal{R}(\mathcal{I}) \subset \mathcal{M}$. Further, by Theorem 3.6, we have that $\mathcal{M}$ is closed under bounded monotone limits and so $\mathcal{M}$ is a local monotone class. By the (local) monotone class theorem (Kallenberg, 2017, Lemma 1.2), $\mathcal{M}$ contains the local monotone ring generated by the $\operatorname{ring} \mathcal{R}(\mathcal{I})$ which is equal to $\mathcal{S}_{b}$. Hence $\left(M(C), M\left(B_{2}\right), \ldots, M\left(B_{m}\right)\right)$ is NA for all $C \in \mathcal{S}_{b}$ such that $C$ is disjoint from $B_{2}, \ldots, B_{m}$. Repeating this argument, we can derive the asserted property for all disjoint $B_{1}, \ldots, B_{m} \in \mathcal{S}_{b}$.

Proof of Theorem 4.4: Again, we shall prove only for NA and the proof for the PA case follows similarly. The 'only if' part is trivial and so we shall prove the 'if' part. Fix a pair $f, g$ of bounded non-decreasing functions that are measurable with respect to disjoint measurable subsets of $\mathbb{S}$, say $A, B$. Using (4.2), define on $\mathbb{G}$ two measurable functions $\tilde{f}:=f \circ \gamma^{-1}$, and $\tilde{g}:=g \circ \gamma^{-1}$. It is not hard to prove (by a monotone class argument for instance) that $\gamma^{-1}$ is non-decreasing, so

that $\tilde{f}$ and $\tilde{g}$ are non-decreasing. Define $X^{A}:=\left(M\left(I_{n} \cap A\right)\right)_{n \geq 1}=\gamma\left(M_{A}\right)$ and $X^{B}:=\left(M\left(I_{n} \cap B\right)\right)_{n \geq 1}$. Suppose we can show that

$$
\mathbb{E}\left[\tilde{f}\left(X^{A}\right) \tilde{g}\left(X^{B}\right)\right] \leq \mathbb{E}\left[\tilde{f}\left(X^{A}\right)\right] \mathbb{E}\left[\tilde{g}\left(X^{B}\right)\right]
$$

Then we would obtain that

$$
\begin{aligned}
\mathbb{E}[f(M) g(M)] & =\mathbb{E}\left[f\left(M_{A}\right) g\left(M_{B}\right)\right]=\mathbb{E}\left[\tilde{f}\left(X^{A}\right) \tilde{g}\left(X^{B}\right)\right] \\
& \leq \mathbb{E}\left[\tilde{f}\left(X^{A}\right) \mathbb{E}\left[\tilde{g}\left(X^{B}\right)\right]=\mathbb{E}[f(M)] \mathbb{E}[g(M)]\right.
\end{aligned}
$$


as desired.

It remains to prove (4.4) for arbitrary bounded non-decreasing measurable functions $\tilde{f}$ and $\tilde{g}$. To this end, we take $m \in \mathbb{Z}_{+}$and show that

$$
\begin{aligned}
& \mathbb{E}\left[h_{1}\left(M\left(I_{k} \cap A\right)_{k=1}^{m}\right) h_{2}\left(M\left(I_{k} \cap B\right)_{k=1}^{m}\right)\right] \\
& \leq \mathbb{E}\left[h_{1}\left(M\left(I_{k} \cap A\right)_{k=1}^{m}\right)\right] \mathbb{E}\left[h_{2}\left(M\left(I_{k} \cap B\right)_{k=1}^{m}\right)\right],
\end{aligned}
$$

for all bounded non-decreasing measurable functions $h_{1}: \mathbb{R}_{+}^{m} \rightarrow \mathbb{R}$ and $h_{2}: \mathbb{R}_{+}^{m} \rightarrow$ $\mathbb{R}$. There exist $l \in \mathbb{Z}_{+}$and disjoint sets $I_{i}^{\prime} \in \mathcal{I}, i \in\{1, \ldots, l\}$, such that $I_{j}=\cup_{i \in J_{j}} I_{i}^{\prime}$ for $j \in\{1, \ldots, m\}$, where $J_{j} \subset\{1, \ldots, l\}$ for all $j$. Defining

$$
h_{1}^{\prime}\left(\left(x_{i}\right)_{i=1}^{l}\right):=h_{1}\left(\sum_{i \in J_{1}} x_{i}, \ldots, \sum_{i \in J_{m}} x_{i}\right)
$$

we observe that $h_{1}^{\prime}$ is coordinatewise non-decreasing as $h_{1}$ is coordinatewise nondecreasing. Similarly, we can define $h_{2}^{\prime}$. By disjointness of $I_{1}^{\prime}, \ldots, I_{l}^{\prime}$ and $A \cap B=\emptyset$, we have by assumption and Lemma 4.5 that the random vector

$$
\left(M\left(I_{1}^{\prime} \cap A\right), \ldots, M\left(I_{l}^{\prime} \cap A\right), M\left(I_{1}^{\prime} \cap B\right), \ldots, M\left(I_{l}^{\prime} \cap B\right)\right)
$$

is negatively associated. Therefore

$$
\begin{aligned}
& \mathbb{E}\left[h _ { 1 } ^ { \prime } \left(\left(M\left(I_{i}^{\prime} \cap A\right)_{i=1}^{l}\right) h_{2}^{\prime}\left(\left(M\left(I_{i}^{\prime} \cap B\right)_{i=1}^{l}\right)\right]\right.\right. \\
\leq & \mathbb{E}\left[h _ { 1 } ^ { \prime } ( ( M ( I _ { i } ^ { \prime } \cap A ) _ { i = 1 } ^ { l } ) ] \mathbb { E } \left[h_{2}^{\prime}\left(\left(M\left(I_{i}^{\prime} \cap B\right)_{i=1}^{l}\right)\right] .\right.\right.
\end{aligned}
$$

By definition of $h_{1}^{\prime}, h_{2}^{\prime}$, the above inequality is equivalent to the inequality (4.5). Now using Corollary 3.4, we obtain (4.4) as required to complete the proof.

We use $\stackrel{d}{\rightarrow}$ to denote weak convergence of random measures as well.

Theorem 4.6. Suppose $M_{n}, n \geq 1$ are associated random measures on a Polish space $\mathbb{S}$ and $M_{n} \stackrel{d}{\rightarrow} M$. Then $M$ is also associated as a random measure in the same positive or negative way as the elements of the sequence.

Proof: As before, we prove only the NA case and the PA case follows analogously. Define $\mathcal{S}_{M}:=\left\{B \in \mathcal{S}_{b}: \mathbb{E}[M(\partial B)]=0\right\}$ where $\partial B$ is the boundary of a set $B$. Since $M_{n} \stackrel{d}{\rightarrow} M$, we have that $\left(M_{n}\left(B_{1}\right), \ldots, M_{n}\left(B_{k}\right)\right) \stackrel{d}{\rightarrow}\left(M\left(B_{1}\right), \ldots, M\left(B_{k}\right)\right)$ for all $B_{1}, \ldots, B_{k} \in \mathcal{S}_{M}$ (see Kallenberg, 2017, Theorem 4.11). Thus by Theorem 3.6, we have that $\left(M\left(B_{1}\right), \ldots, M\left(B_{k}\right)\right)$ is NA for all pairwise disjoint $B_{1}, \ldots, B_{k} \in \mathcal{S}_{M}$. Since $\mathcal{S}_{M}$ is a dissecting ring and there exists $\mathcal{I} \subset \mathcal{S}_{M}$, a countable, topological, dissecting, semi-ring generating the $\sigma$-field $\mathcal{S}$ (Kallenberg, 2017, Lemma 1.9), by Theorem 4.4, we have that $M$ is a NA random measure.

\section{Examples}

In this section, we recall some known and give some new examples of associated random measures and fields. As mentioned in the introduction, showing many of these examples are associated in the strong sense as in Definitions 3.1, 4.2 and (4.1) shall require our Theorems 3.3, 3.6, 4.4 and 4.6. In an appendix, we recall some classical results related directly to applied probability models. We are not aware of many examples of NA random fields. 


\subsection{Associated random fields.}

Example 5.1. (Gaussian random measures and fields.) Suppose that $M$ is a Gaussian random measure on $\mathbb{S}$ such that $\operatorname{Cov}[M(A), M(B)] \leq 0$ for $A$ and $B$ disjoint. Then from Joag-Dev and Proschan (1983, Section 3.4) and our Theorem 3.3, we have that $M$ is NA. A simple special case is when $\mathbb{S}$ is a discrete set and $M:=\sum_{s \in \mathbb{S}} X_{s} \delta_{s}$ where $\mathbf{X}:=\left(X_{s}\right)_{s \in \mathbb{S}}$ is a Gaussian random field such that $\operatorname{Cov}\left[X_{s}, X_{t}\right] \leq 0$ for all $s \neq t$, which implies that $\mathbf{X}$ is NA. Similarly, by Pitt (1982) and our Theorem 3.3, the condition $\mathbb{C o v}\left(X_{s}, X_{t}\right) \geq 0, s, t \in \mathbb{S}$, is necessary and sufficient for the random measure $M$ to be PA.

Example 5.2. (Dirichlet sequences) Let $\alpha_{n} \geq 0, n \in \mathbb{Z}_{+}$, be such that $\alpha:=\sum_{n=1}^{\infty} \alpha_{n}$ is positive and finite. Let $X_{1}, X_{2}, \ldots$ be independent Gamma distributed random variables with shape parameters $\alpha_{1}, \alpha_{2}, \ldots$ and scale parameter 1 . Then $X:=$ $\sum_{n=1}^{\infty} X_{n}$ has a Gamma distribution with shape parameter $\alpha$ and $\left(X^{-1} X_{n}\right)_{n \geq 1}$ is NA. To see the latter we first assume that there exists $m \in \mathbb{Z}_{+}$such that $\alpha_{n}=0$ for $n>m$. Then $X^{-1}\left(X_{1}, \ldots, X_{m}\right)$ has a Dirichlet distribution with parameter $\left(\alpha_{1}, \ldots, \alpha_{m}\right)$. Moreover, since the latter random vector is independent of $X$, we obtain from Joag-Dev and Proschan (1983, Theorem 2.8) that it is NA. Since $\left(X^{-1} X_{n}\right)_{n \geq 1}$ can be almost surely approximated by the sequences

$$
\left(X_{1}+\cdots+X_{m}\right)^{-1}\left(X_{1}, \ldots, X_{m}, 0,0, \ldots\right), \quad m \geq 1,
$$

Theorem 3.6 shows that $\left(X^{-1} X_{n}\right)_{n \geq 1}$ is NA.

Example 5.3. (Markov stochastically monotone, up-down processes, Liggett, 2005, Szekli, 1995, Section 3.8, Theorem A) Let $\mathbf{X}=(X(t), t \geq 0)$ be a time homogeneous Markov Feller process with values in a partially ordered Polish space $\mathbb{X}$ with generator $A$. If $\mathbf{X}$ is stochastically monotone and up-down (i.e. $A f g \geq$ $f A g+g A f$, for non-decreasing $f, g)$ and $X(0)$ is positively associated then $\mathbf{X}$ is PA, i.e. $\left(X\left(t_{1}\right), \ldots, X\left(t_{n}\right)\right)$ is PA as a random element of $\mathbb{X}^{n}$, for all $t_{1}<\ldots<t_{n}$, $n \in \mathbb{Z}_{+}$, and the invariant (stationary) distribution of $\mathbf{X}$ is PA (if it exists). Using our results the PA property can be extended to infinite sequences. In this class of Markov processes many particle systems (attractive) and generalized birth and death processes are included.

Example 5.4. (Random integrals) Let $I$ be a countable index set and let $\mathbb{S}$ be a partially ordered Polish space. Suppose that $f_{y}: \mathbb{S} \rightarrow \mathbb{R}_{+}, y \in I$, is a family of measurable functions and that $M$ is a random measure on $\mathbb{S}$. Define a random field $\mathbf{X}=\left(X_{y}\right)_{y \in I}$ by

$$
X_{y}:=\int f_{y}(x) M(d x), \quad y \in I .
$$

If $M$ is $\mathrm{PA}$, then so is $\mathbf{X}$. For simple functions $f_{y}$, this is straightforward from the definition of PA and then for arbitrary functions one can use the standard approximation along with our weak convergence result (Theorem 3.6).

\subsection{Associated random measures.}

Example 5.5. (Poisson process) Let $\Pi$ be a Poisson process on a Polish space $\mathbb{S}$ with a locally finite intensity measure $\lambda$. By complete independence, $\Pi$ is NA. It was stated in Roy (1990) (referring to the author's PhD-thesis) that $\Pi$ is PA. We refer to Last and Penrose (2018, Theorem 20.4) for a general version of this result. In 
the percolation literature this is better known as the Harris-FKG inequality (see Harris, 1960 and Fortuin et al., 1971). If $\lambda$ is diffuse, then Last and Penrose (2018, Theorem 6.14) shows that a Poisson process is the only simple point process with intensity measure $\lambda$ which is both PA and NA.

Example 5.6. (Mixed Poisson process) Let $\lambda$ be a locally finite measure on a Polish space $\mathbb{S}$. Let $X \geq 0$ be a random variable and suppose that $\Phi$ is a point process on $\mathbb{S}$ such that a.s. $\mathbb{P}(\Phi \in \cdot \mid X)=\Pi_{X \lambda}$, where, for a given locally finite measure $\nu$ on $\mathbb{S}, \Pi_{\nu}$ denotes the distribution of a Poisson process with intensity measure $\nu$. Then $\Phi$ is known as a mixed Poisson process. We show that $\Phi$ is PA; see Georgii and Küneth (1997, Example 2.1). Let $f, g: \mathbb{S} \rightarrow \mathbb{R}$ be measurable bounded and non-decreasing. By conditioning and Example 5.5

$$
\mathbb{E} f(\Phi) g(\Phi) \geq \mathbb{E}[\mathbb{E}[f(\Phi) \mid X] \mathbb{E}[g(\Phi) \mid X]]=\mathbb{E}[\tilde{f}(X) \tilde{g}(X)],
$$

where $\tilde{f}(x):=\int f(\mu) \Pi_{x \lambda}(d \mu), x \geq 0$, and the function $\tilde{g}$ is defined similarly. A simple thinning argument (see e.g. Last and Penrose, 2018, Corollary 5.9) shows that $\tilde{f}$ and $\tilde{g}$ are non-decreasing. Since a single random variable in a totally ordered space is PA (Lindqvist, 1988, Theorem 3.4), we obtain that

$$
\mathbb{E}[\tilde{f}(X) \tilde{g}(X)] \geq \mathbb{E}[\tilde{f}(X)] \mathbb{E}[\tilde{g}(X)]=\mathbb{E}[f(\Phi)] \mathbb{E}[g(\Phi)],
$$

as asserted.

Example 5.7. (Cox processes) Let $\Lambda$ be a random measure on a Polish space $\mathbb{S}$ and let $\Phi$ be a point process on $\mathbb{S}$ such that a.s. $\mathbb{P}(\Phi \in \cdot \mid \Lambda)=\Pi_{\Lambda}$. Then $\Phi$ is known as a Cox process. We show that if $\Lambda$ is associated, then so is $\Phi$. Assume first that $\Lambda$ is PA. Let $f, g: \mathbb{S} \rightarrow \mathbb{R}$ be measurable bounded and non-decreasing. Since Poisson processes are PA we have similarly as in Example 5.6 that $\mathbb{E}[f(\Phi) g(\Phi)] \geq$ $\mathbb{E}[\tilde{f}(\Lambda) \tilde{g}(\Lambda)]$, where $\tilde{f}(\nu):=\int f(\mu) \Pi_{\nu}(d \mu), \nu \in \mathbf{M}(\mathbb{S})$, and $\tilde{g}$ is defined similarly. By the thinning properties of Poisson processes the (measurable) functions $\tilde{f}$ and $\tilde{g}$ are non-decreasing. Hence $\mathbb{E}[\tilde{f}(\Lambda) \tilde{g}(\Lambda)] \geq \mathbb{E}[f(\Phi)] \mathbb{E}[g(\Phi)]$ and $\Phi$ is PA. Assume now that $\Lambda$ is NA and that $f$ and $g$ are measurable with respect to disjoint measurable subsets of $\mathbb{S}$. Using in the above calculation the complete independence of a Poisson process instead of PA, and the fact that for each measurable set $A$ the restriction $\Phi_{A}$ is Cox with directing measure $\Lambda_{A}$, we obtain that $\Phi$ is NA. The PA case of this example generalizes Example 5.6 and, in fact, Theorem 5.5 in Burton and Waymire (1985). The NA case might be new, at least in this generality. Note that our strong (functional) definition of association has been crucial for the above arguments.

Example 5.8. (Permanental point processes) Assume that $\mathbb{S}$ is a locally compact separable metric space and let $\mathbf{X}=\left(X_{s}\right)_{s \in \mathbb{S}}$ be a Gaussian random field. It was shown in Eisenbaum (2014) that the finite-dimensional distributions of $\left(X_{s}^{2}\right)_{s \in \mathbb{S}}$ are PA iff they are infinitely divisible. Assume this is the case and that moreover, $\mathbf{X}$ has continuous sample paths. Let $\mu$ be a locally finite measure on $\mathbb{S}$ and define $\Lambda:=\int \mathbf{1}\{s \in \cdot\} X_{s}^{2} \mu(d s)$. It can be shown as in Example 5.12 below that the random measure $\Lambda$ is PA. By Example 5.7, a Cox process $\Phi$ directed by $\Lambda$ is PA. Such a $\Phi$ is a special case of a (1/2)-permanental process; see e.g. Last and Penrose (2018, Chapter 14). More generally, we may consider $k$ i.i.d. infinitely divisible Gaussian random fields $\mathbf{X}^{1}, \ldots, \mathbf{X}^{k}$ as above and define $\Lambda:=\int \mathbf{1}\{s \in \cdot\} Y_{s} \mu(d s)$, where $Y_{s}:=\left(X_{s}^{1}\right)^{2}+\cdots+\left(X_{s}^{k}\right)^{2}$. By a basic property of association the field $\left(Y_{s}\right)_{s \in \mathbb{S}}$ 
is again PA, so that a Cox process $\Phi$ with directing measure $\Lambda$ is PA as well. Such a $\Phi$ is $k / 2$-permanental; see again Last and Penrose (2018, Chapter 14).

Example 5.9. (Determinantal point processes, Lyons, 2014, Theorem 3.7) Let $\lambda$ be a Radon measure on a locally compact Polish space $\mathbb{X}$. Let $\mathrm{K}$ be a locally trace-class positive contraction on $L_{2}(\mathbb{X}, \lambda)$. The determinantal point process defined by $\mathrm{K}$ is NA as a random measure. Well known examples of determinantal point processes are descents in random sequences (Borodin et al., 2010), non-intersecting random walks (Johansson, 2004), edges in random spanning trees (Burton and Pemantle, 1993) and the finite and infinite Ginibre ensemble (Ginibre, 1965, see also Section 6).

Example 5.10. (Mixed sampled point processes, Last and Szekli, 2019, Theorem 3.3) Suppose that $N:=\sum_{i=1}^{\tau} \delta_{X_{i}}$, where $X_{i}$ are i.i.d. on a Polish space $\mathbb{X}$ and $\tau \in$ $\mathbb{Z}_{+} \cup\{0\}$ is independent of $\left(X_{i}\right)_{i \geq 1}$. This is called as a mixed sampled point process; see also Last and Penrose (2018). If $\tau$ has an ultra log-concave distribution, then $N$ is NA as a random point process. This example can be immediately extended to the case of random measures $M:=\sum_{i=1}^{\tau} W_{i} \delta_{X_{i}}$, for an independent iid sequence $\left(W_{i}\right)$ of positive random variables. Such random measures belong to the class of random measures described in the next example.

Example 5.11. (Independently-weighted point processes) Suppose that $N=$ $\left\{X_{i}\right\}_{i \geq 1}$ is a NA point process on $\mathbb{S}$ and $\left(W_{i}\right)_{i \geq 1}$ is an independent but possibly position dependent marking of $N$ with non-negative marks (see Last and Penrose, 2018, Section 5.2 for more details). In other words, given $N$, let $\left(W_{i}\right)$ be independent random variables chosen as per distribution $K\left(X_{i},.\right)$, where $K(x, d w)$ is the probability kernel generating the independent marking. Define the random measure $M:=\sum_{i} W_{i} \delta_{X_{i}}$. Clearly we have a.s. that $\mathbb{P}(M \in \cdot \mid N)=K^{*}(N, \cdot)$ for a suitably defined probability kernel $K^{*}$. Suppose that $f, g: \mathbf{M}(\mathbb{S}) \rightarrow \mathbb{R}$ are bounded measurable and non-decreasing. Assume also that there exists a measurable $A \subset \mathbb{S}$ such that $f$ is measurable w.r.t. $A$ and $g$ is measurable w.r.t. $A^{c}$. Since $M_{A}$ and $M_{A^{c}}$ are conditionally independent given $N$, we have a.s. that $\mathbb{E}[f(M) g(M)]=\mathbb{E}\left[[\mathbb{E}[f(M) \mid N] \mathbb{E}[g(M) \mid N]]\right.$. We can define $K^{*}$ in such a way that $\int f(\nu) K^{*}(\mu, d \nu)$ and $\int g(\nu) K^{*}(\mu, d \nu)$ are increasing in $\mu$. Therefore $M$ is NA.

Example 5.12. (Integral of random fields) Suppose $\mathbb{X}$ is a Polish space with a locally finite measure $\mu$ and $\mathbf{X}:=(X(x))_{x \in \mathbb{X}}$ is a $\mathbb{Y}$-valued continuous random field where $\mathbb{Y}$ is a POP space. Assume that $(X(x))_{x \in I}$ is NA for any finite $I \subset \mathbb{X}$. Let $f: \mathbb{Y} \rightarrow \mathbb{R}_{+}$ be an increasing and continuous function. Then we have that the random measure $M(A):=\int_{A} f(X(x)) \mu(\mathrm{d} x), A \in \mathcal{S}$, is a NA random measure. This can be proved as follows. Easily we have that $(f(X(x)))_{x \in I}$ is NA for any finite $I \subset \mathbb{X}$. Now, we approximate $M(A)$ for any $A \in \mathcal{S}_{b}$ as follows. Let $\left\{x_{n}\right\}_{n \geq 1}$ be a countable dense set of $\mathbb{X}$ and $B_{n}^{k}:=B_{x_{n}}\left(2^{-k}\right) \backslash\left(\cup_{m=1}^{n-1} B_{x_{m}}\left(2^{-k}\right)\right)$. Choose $y_{n}^{k} \in B_{n}^{k}$ for all $n, k$. Define

$$
M_{k}(A):=\int_{A} \sum_{n=1}^{\infty} \mathbf{1}\left\{x \in B_{n}^{k}\right\} \mathbf{1}\left\{y_{n}^{k} \in A\right\} f\left(X\left(y_{n}^{k}\right)\right) \mu(\mathrm{d} x) .
$$

Observe that $M_{k}(A)$ is an increasing function of $\left\{f\left(X\left(y_{n}^{k}\right)\right)\right\}_{y_{n}^{k} \in A}$ and by Theorem 3.3, $\left\{f\left(X\left(y_{n}^{k}\right)\right)\right\}_{y_{n}^{k} \in A}$ is a NA random field. Thus for disjoint bounded sets $A_{1}, \ldots, A_{m}$, since $M_{k}\left(A_{i}\right)$ 's are increasing functions of disjoint collection of $f\left(X\left(y_{n}^{k}\right)\right)$, we have that $\left(M_{k}\left(A_{1}\right), \ldots, M_{k}\left(A_{m}\right)\right)$ is NA. By continuity of $f, \mathbf{X}$ and boundedness of $A_{i}$ 's, we can use the dominated convergence theorem to show that 
for all $1 \leq i \leq m, M_{k}\left(A_{i}\right) \rightarrow M\left(A_{i}\right)$ a.s. as $k \rightarrow \infty$. Now, by using Theorem 3.6, we have that $\left(M\left(A_{1}\right), \ldots, M\left(A_{m}\right)\right)$ is NA for disjoint bounded sets $A_{1}, \ldots, A_{m}$ and hence $M$ is NA by Theorem 4.4.

Example 5.13. (Dirichlet process) Let $\lambda$ be a measure on $\mathbb{S}$ such that $0<\lambda(S)<\infty$. A random measure $M$ on $\mathbb{S}$ is called a Dirichlet process (Ferguson, 1973; Last and Penrose, 2018) with parameter measure $\lambda$ if $\left(M\left(B_{1}\right), \ldots, M\left(B_{n}\right)\right)$ has a Dirichlet distribution with parameter $\left(\lambda\left(B_{1}\right), \ldots, \lambda\left(B_{n}\right)\right)$, whenever $B_{1}, \ldots, B_{n}, n \geq 1$, form a measurable partition of $\mathbb{S}$. By Example 5.2, a Dirichlet process is NA. Note that the NA property of Dirichlet sequences is in accordance with Theorem 4.4.

Example 5.14. (Infinitely divisible random measures, Burton and Waymire, 1986, Evans, 1990) Suppose that $M$ is a random measure on a Polish space $\mathbb{S}$ which is infinitely divisible. This means that for any $n \in \mathbb{Z}_{+}$, there exist independent identically distributed random measures $M_{1}, \ldots, M_{n}$ on $\mathbb{S}$ such that $M$ has the same distribution as $M_{1}+\cdots+M_{n}$. It was shown in Burton and Waymire (1986) and Evans (1990) that $M$ is PA. We give here a short proof of this result which does, moreover, not require $\mathbb{S}$ to be locally compact. By a classical point process result (see e.g. Kallenberg, 2017, Theorem 3.20) there exists a Poisson process $\Phi$ on $\mathbf{M}(\mathbb{S})$ and a measure $\lambda \in \mathbf{M}(\mathbb{S})$ such that $M=\lambda+\int \mu \Phi(d \mu)$ holds a.s. Taking measurable bounded and non-decreasing functions $f, g: \mathbf{M}(\mathbb{S}) \rightarrow \mathbb{R}$, we obtain that

$$
\mathbb{E}[f(M) g(M)]=\mathbb{E}[\tilde{f}(\Phi) \tilde{g}(\Phi)],
$$

where the function $\tilde{f}$ (and similarly $\tilde{g}$ ) is defined as follows. Given a locally finite counting measure $\varphi$ on $\mathbf{M}(\mathbb{S})$ we set $\tilde{f}(\varphi):=f\left(\lambda+\int \mu \varphi(d \mu)\right)$ whenever the measure $\int \mu \varphi(d \mu)$ is locally finite. Otherwise we set $\tilde{f}(\varphi):=c$, where $c$ is an upper bound of $f$. Since $\tilde{f}$ and $\tilde{g}$ are non-decreasing we can apply the PA property of $\Phi$ (see Example 5.5) to conclude that $\mathbb{E}[\tilde{f}(\Phi) \tilde{g}(\Phi)] \geq \mathbb{E}[\tilde{f}(\Phi)] \mathbb{E}[\tilde{g}(\Phi)]=\mathbb{E}[f(M)] \mathbb{E}[g(M)]$, as asserted.

Example 5.15. (Poisson cluster random measure) Suppose that $N=\sum_{i=1}^{\tau} \delta_{\xi_{i}}$ is a Poisson process on a Polish space $\mathbb{S}$. Let $\left(M_{i}, i \geq 1\right)$ be an i.i.d. sequence of random measures on $\mathbb{S}$, independent of $N$. Assume that

$$
\iint \min (\mu(B+x), 1) \mathbb{P}\left(M_{1} \in d \mu\right) \mathbb{E}[\Phi](d x)<\infty
$$

for all bounded Borel sets $B \subset \mathbb{S}$. By Kallenberg (2017, Theorem 3.20) the random measure $M$ defined by $M(B)=\sum_{i=1}^{\tau} M_{i}\left(B+\xi_{i}\right), B \in \mathcal{S}$, is infinitely divisible. Example 5.14 shows that $M$ is PA.

Example 5.16. (Self-exciting point processes on the real axis, Kwieciński and Szekli, 1996, Theorem 4.2) Let $N$ be a point process on $\mathbb{R}_{+}$admitting stochastic intensity with respect to its internal filtration. If $N$ is a positively self-exciting w.r.t. $\prec$, then $N$ is positively associated w.r.t. $\prec$, whenever $\prec$ denotes one of the three orderings of point processes introduced there. In particular renewal processes with inter-point distribution which has decreasing failure rate (DFR) are PA as random measures.

Example 5.17. (Area interaction process) Let $\mathbb{S}$ be a compact subset of $\mathbb{R}^{d}$ equipped with the Euclidean distance. Let $\beta>0$ and let $\Pi_{\beta}$ be the distribution of a Poisson process with intensity measure $\beta \lambda_{d}$ restricted to $\mathbb{S}$, where $\lambda_{d}$ denotes Lebesgue measure on $\mathbb{R}^{d}$. Fix a number $r>0$ and define $U(\mu):=\cup_{x \in \mu} B(x, r), \mu \in \mathbf{N}(\mathbb{S})$, 
where $B(x, r)$ is the Euclidean ball with radius $r$ centred at $x$. Suppose that $\Phi$ is a point process on $\mathbb{S}$ whose distribution is absolutely continuous w.r.t. $\Pi_{\beta}$, with density proportional to $p(\mu)=e^{-\alpha \lambda_{d}(U(\mu))}, \mu \in \mathbf{N}(\mathbb{S})$, where $\alpha>0$ is another parameter. Example 2.3 in Georgii and Küneth (1997) shows the finite dimensional distributions of $\Phi$ are positively associated thus using our Theorem 4.4 we conclude that $\Phi$ is PA. In fact, the latter example covers a more general class of finite Gibbs processes (of Widom-Rowlinson type) which are PA.

Example 5.18. (Exclusion processes) The symmetric exclusion process on a countable set $S$ is the Markov process $\left(X_{t}, t \geq 0\right)$ on the state space $E=\{0,1\}^{S}$ with the formal generator

$$
L f(\eta)=\sum_{x, y: \eta(x)=1, \eta(y)=0} p(x, y)\left[f\left(\eta_{x, y}\right)-f(\eta)\right], \eta \in E,
$$

where $\eta_{x, y}$ is the configuration obtained from $\eta$ by interchanging the coordinates $\eta(x)$ and $\eta(y)$. Here $p(x, y)=p(y, x)$ are the transition probabilities for a symmetric, irreducible, Markov chain on $S$. For background on this process, see Chapter VIII of Liggett (2005). Let

$$
\mathcal{H}=\left\{\alpha: S \rightarrow[0,1], \sum_{y} p(x, y) \alpha(y)=\alpha(x) \forall x\right\},
$$

and for $\alpha \in \mathcal{H}$, let $\nu_{\alpha}$ be the product measure with marginals $\nu_{\alpha}(\eta: \eta(x)=1)=$ $\alpha(x)$. Then the limiting distribution as $t \rightarrow \infty$ of the process $\left(X_{t}\right)$ exists if the initial distribution of $X_{0}$ is $\nu_{\alpha}$; call it $\mu_{\alpha}$. It is known from Borcea et al. (2009) that for $\mu_{\alpha}$ the finite dimensional distributions are negatively associated and using our Theorem 3.3 we have that $\mu_{\alpha}$ is NA.

\section{Appendix}

In order to make the list of examples more complete we recall some classical results related directly to applied probability models.

a) (Non-Gaussian infinitely divisible random vectors, Samorodnitsky, 1995) Let X be an infinitely divisible random vector with Lévy measure $\nu$ which is concentrated on the positive $\left(\mathbb{R}_{+}\right)^{d}$ and the negative $\left(\mathbb{R}_{-}\right)^{d}$ quadrants of $\mathbb{R}^{d}$ then $\mathbf{X}$ is $\mathrm{PA}$. This condition is not necessary in general but it is for some sub-classes of infdiv vectors.

b) (Max infinitely divisible random vectors, Resnick, 1988) A random vector $\mathbf{X}$ is max-infinitely divisible if for every $n \in \mathbb{Z}_{+}$there exist i.i.d. random vectors $\mathbf{X}_{n 1}, \mathbf{X}_{n 2}, \ldots, \mathbf{X}_{n n}$ such that $\mathbf{X}$ is equal in distribution to $\max \left(\mathbf{X}_{n 1}, \mathbf{X}_{n 2}, \ldots, \mathbf{X}_{n n}\right)$. Every max-infinitely-divisible random vector $\mathbf{X}$ is PA.

c) (Karlin and Rinott, 1980). If the distribution of a vector $\mathbf{X}$ has density $f$ such that $f(\mathbf{x} \vee \mathbf{y}) f(\mathbf{x} \wedge \mathbf{y}) \geq f(\mathbf{x}) f(\mathbf{y})$, for all $\mathbf{x}, \mathbf{y} \in \mathbb{R}^{n}$ it is called multivariate totally positive of order $2\left(M T P_{2}\right)$. An $M T P_{2}$, random vector $\mathbf{X}$ induces an $\mathrm{PA}$ set of random variables (FKG inequalities). The following special cases are classical $M T P_{2}$ densities: (i) the negative multinomial discrete density; (ii) $\mathbf{X}$ is normally distributed with mean zero and the covariance matrix $\Sigma$ is $M T P_{2}$ if and only $-\Sigma^{-1}$ exhibits nonnegative off-diagonal elements (that is $\Sigma^{-1}$ is so called M-matrix or Leontief matrix); (iii) the density of the eigenvalues of certain Wishart random matrices; (iv) the density of multivariate logistic distribution; 
(v) the density of the multivariate gamma distribution; (vi) the density of the multivariate Cauchy distribution.

d) (Virtual waiting time process, Kwieciński and Szekli, 1996, Proposition 5.1) Suppose that a marked point process $N$ feeding a single-server queue is positively associated as random measure. Then the processes of the virtual waiting time and of the number of customers in the system are PA as random fields.

e) (M-infinitely divisible random sets, Karłowska-Pik and Schreiber, 2008, Theorem 2.1) If M-infinitely-divisible convex compact random set $\mathbf{X}$ has no Gaussian summand and its Lévy measure concentrates on the family of sets containing the origin, then $\mathbf{X}$ is $\mathrm{PA}$ as a random element of the space of closed subsets of $\mathbb{R}^{d}$ equipped with the Fell topology. Similarly, every union-infinitely-divisible random closed set is PA.

f) (Sojourn times on quasi overtake-free paths in queueing networks, Daduna and Szekli, 2004, Theorem 4.2 and 6.4). The vector of a test customer's successive sojourn times on a quasi overtake-free path in a closed Gordon-Newell queueing network is negatively associated. In particular, the vector of a test customer's successive sojourn times in a cycle is NA.

g) (Queueing networks, Szekli, 1995, Section 3.8, Theorem E) Let $(X(t), t \geq 0)$ denote the joint queue length process of an irreducible Gordon-Newell network with Markovian routing and queue-length dependent non-decreasing service rates, which acts in equilibrium. Then for each $t \geq 0, X(t)$ is NA.

h) (Eigenvalues of random matrices, Ginibre, 1965) Let $M$ be a random matrix obtained by drawing every entry independently from the complex normal distribution. This is the complex Ginibre ensemble. The eigenvalues of $M$, which form a finite subset of the complex plane define a NA point process (which is determinantal). If a Hermitian matrix is generated in the corresponding way, drawing each diagonal entry from the normal distribution and each pair of off-diagonal entries from the complex normal distribution, then we obtain the Gaussian unitary ensemble, and the eigenvalues are now a NA (determinantal) point process on the real line.

i) (Van den Berg and Kesten (BK) inequality) Let $E=\{0,1\}^{n}$, and $[n]:=$ $\{1, \ldots, n\}$. For $\eta \in E$ and $I \subset[n]$, let $\eta_{I}$ denote the tuple $\left(\eta_{i}, i \in I\right)$. By $[\eta]_{I}:=\left\{\alpha \in E: \alpha_{I}=\eta_{I}\right\}$ we denote the set of all elements of $E$ that agree with $\eta$ on $I . A \square B$ defines the event that $\mathrm{A}$ and $\mathrm{B}$ occur disjointly, that is

$$
A \square B=\left\{\eta \in E: \exists \text { disjoint } K, L \subset[n],[\eta]_{K} \subset A,[\eta]_{L} \subset B\right\} .
$$

An event $A \subseteq E$ is said to be increasing if $\eta^{\prime} \in A$ whenever $\eta \in A$ and $\eta^{\prime} \geq \eta$ coordinatewise. A probability measure $P$ on $E$ is BK if for all increasing $A, B$, $P(A \square B) \leq P(A) P(B)$. It is known that if $P$ is BK then it is NA but NA does not imply BK, see van den Berg and Jonasson (2012).

j) (Distributions on vertices of polytopes in $\mathbb{R}^{n}$, Peres et al., 2017) For a Gaussian random walk in a polytope that starts at a point inside and continues until it gets absorbed at a vertex the probability distribution induced on the vertices by this random walk is NA for matroid polytopes. Such distributions are highly sought after in randomized algorithms as they imply concentration properties.

k) (Random-cluster model, Grimmett, 2006) The random cluster measure $\phi_{p, q}$ is PA for all $p \in[0,1], q \in[1, \infty)$ with free or wired boundary conditions. For any other boundary condition, the limit random cluster measures and extreme (tail trivial) DLR random cluster measures are PA for all $p \in[0,1], q \in[1, \infty)$ (see 
Grimmett, 2006, Theorems 4.17 and 4.37). It is one of the important conjectures in statistical physics that $\phi_{p, q}$ satisfies some form of negative dependence for $p \in$ $[0,1], q \in(0,1)$. From our Theorem 3.6, this conjecture boils down to showing a suitable negative dependence property for the finite-volume case. However, this is shown in certain special cases of the $q \downarrow 0$ limit (see Grimmett, 2006, Section $3.9)$.

1) (Conditional distributions, $\mathrm{Hu}$ and $\mathrm{Hu}, 1999)$ Let $\mathbf{X}=\left(X_{1}, X_{2}, \ldots, X_{n}\right)$ be a random vector of $n$ iid rv with a continuous distribution. Then $\left[\mathbf{X} \mid X_{\left(k_{1}\right)}=\right.$ $\left.s_{1}, X_{\left(k_{2}\right)}=s_{2}, \ldots, X_{\left(k_{r}\right)}=s_{r}\right]$ is NA for $1 \leq k_{1}<k_{2}<\ldots<k_{r} \leq n$ and $s_{1}<s_{2}<\ldots<s_{r}$, where $X_{(1)} \leq \ldots \leq X_{(n)}$ are the order statistics of $\mathbf{X}$. If $\mathbf{X}$ is a random vector of $n$ iid rv with PF2 densities or mass functions then $\left[\mathbf{X} \mid \sum_{i=1}^{n} X_{i} \in(a, b)\right]$ is NA, where $a<b$. Some special cases of conditional distributions are given in the next example.

m) (Joag-Dev and Proschan, 1983) Random vectors X with the permutation, multinomial, multivariate hypergeometric or Dirichlet distributions are NA. For Dirichlet, see also Example 5.13.

\section{Acknowledgments}

The authors wish to thank an anonymous referee for pointing out errors in the proofs of Proposition 3.3 (now Lemma 3.5) and Theorem 3.6 of the earlier version. DY also wishes to thank Bartek Blaszczyszyn and Subhrosekhar Ghosh for some discussions on negatively associated point processes.

\section{References}

Alam, K. and Saxena, K. M. L. Positive dependence in multivariate distributions. Comm. Statist. A-Theory Methods, 10 (12), 1183-1196 (1981). MR623526.

Billingsley, P. Probability and measure. Wiley Series in Probability and Mathematical Statistics. John Wiley \& Sons, Inc., New York, third edition (1995). ISBN 0-471-00710-2. MR1324786.

Borcea, J., Brändén, P., and Liggett, T. M. Negative dependence and the geometry of polynomials. J. Amer. Math. Soc., 22 (2), 521-567 (2009). MR2476782.

Borodin, A., Diaconis, P., and Fulman, J. On adding a list of numbers (and other one-dependent determinantal processes). Bull. Amer. Math. Soc. (N.S.), 47 (4), 639-670 (2010). MR2721041.

Bulinski, A. and Shashkin, A. Limit theorems for associated random fields and related systems, volume 10 of Advanced Series on Statistical Science 85 Applied Probability. World Scientific Publishing Co. Pte. Ltd., Hackensack, NJ (2007). ISBN 978-981-270-940-0; 981-270-940-1. MR2375106.

Bulinski, A. and Spodarev, E. Central limit theorems for weakly dependent random fields. In Stochastic geometry, spatial statistics and random fields, volume 2068 of Lecture Notes in Math., pp. 337-383. Springer, Heidelberg (2013). MR3059652.

Burton, R. and Pemantle, R. Local characteristics, entropy and limit theorems for spanning trees and domino tilings via transfer-impedances. Ann. Probab., 21 (3), 1329-1371 (1993). MR1235419.

Burton, R. and Waymire, E. Scaling limits for associated random measures. Ann. Probab., 13 (4), 1267-1278 (1985). MR806223. 
Burton, R. M. and Waymire, E. The central limit problem for infinitely divisible random measures. In Dependence in probability and statistics (Oberwolfach, 1985), volume 11 of Progr. Probab. Statist., pp. 383-395. Birkhäuser Boston, Boston, MA (1986). MR899999.

Daduna, H. and Szekli, R. On the correlation structure of closed queueing networks. Stoch. Models, 20 (1), 1-29 (2004). MR2036293.

Eisenbaum, N. Characterization of positively correlated squared Gaussian processes. Ann. Probab., 42 (2), 559-575 (2014). MR3178467.

Esary, J. D., Proschan, F., and Walkup, D. W. Association of random variables, with applications. Ann. Math. Statist., 38, 1466-1474 (1967). MR217826.

Evans, S. N. Association and random measures. Probab. Theory Related Fields, 86 (1), 1-19 (1990). MR1061945.

Ferguson, T. S. A Bayesian analysis of some nonparametric problems. Ann. Statist., 1, 209-230 (1973). MR350949.

Fortuin, C. M., Kasteleyn, P. W., and Ginibre, J. Correlation inequalities on some partially ordered sets. Comm. Math. Phys., 22, 89-103 (1971). MR309498.

Georgii, H.-O. and Küneth, T. Stochastic comparison of point random fields. $J$. Appl. Probab., 34 (4), 868-881 (1997). MR1484021.

Georgii, H.-O. and Yoo, H. J. Conditional intensity and Gibbsianness of determinantal point processes. J. Stat. Phys., 118 (1-2), 55-84 (2005). MR2122549.

Ginibre, J. Statistical ensembles of complex, quaternion, and real matrices. $J$. Mathematical Phys., 6, 440-449 (1965). MR173726.

Grimmett, G. The random-cluster model, volume 333 of Grundlehren der Mathematischen Wissenschaften [Fundamental Principles of Mathematical Sciences]. Springer-Verlag, Berlin (2006). ISBN 978-3-540-32890-2; 3-540-32890-4. MR2243761.

Harris, T. E. A lower bound for the critical probability in a certain percolation process. Proc. Cambridge Philos. Soc., 56, 13-20 (1960). MR115221.

Höffding, W. Masstabinvariante Korrelationstheorie. Schr. Math. Inst. u. Inst. Angew. Math. Univ. Berlin, 5, 181-233 (1940). MR0004426.

$\mathrm{Hu}, \mathrm{T}$. and $\mathrm{Hu}$, J. Sufficient conditions for negative association of random variables. Statist. Probab. Lett., 45 (2), 167-173 (1999). MR1718435.

Joag-Dev, K. and Proschan, F. Negative association of random variables, with applications. Ann. Statist., 11 (1), 286-295 (1983). MR684886.

Johansson, K. Determinantal processes with number variance saturation. Comm. Math. Phys., 252 (1-3), 111-148 (2004). MR2103906.

Kallenberg, O. Foundations of modern probability. Probability and its Applications (New York). Springer-Verlag, New York, second edition (2002). ISBN 0-38795313-2. MR1876169.

Kallenberg, O. Random measures, theory and applications, volume 77 of Probability Theory and Stochastic Modelling. Springer, Cham (2017). ISBN 978-3-319-415963; 978-3-319-41598-7. MR3642325.

Kamae, T., Krengel, U., and O'Brien, G. L. Stochastic inequalities on partially ordered spaces. Ann. Probability, 5 (6), 899-912 (1977). MR494447.

Karlin, S. and Rinott, Y. Classes of orderings of measures and related correlation inequalities. I. Multivariate totally positive distributions. J. Multivariate Anal., 10 (4), 467-498 (1980). MR599685. 
Karłowska-Pik, J. and Schreiber, T. Association criteria for $M$-infinitely-divisible and $U$-infinitely-divisible random sets. Probab. Math. Statist., 28 (2), 169-178 (2008). MR2548968.

Kwieciński, A. and Szekli, R. Some monotonicity and dependence properties of self-exciting point processes. Ann. Appl. Probab., 6 (4), 1211-1231 (1996). MR1422983.

Last, G. and Penrose, M. Lectures on the Poisson process, volume 7 of Institute of Mathematical Statistics Textbooks. Cambridge University Press, Cambridge (2018). ISBN 978-1-107-45843-7; 978-1-107-08801-6. MR3791470.

Last, G. and Szekli, R. On negative association of some finite point processes on general state spaces. J. Appl. Probab., 56 (1), 139-152 (2019). MR3981150.

Lehmann, E. L. Some concepts of dependence. Ann. Math. Statist., 37, 1137-1153 (1966). MR202228.

Liggett, T. M. Interacting particle systems. Classics in Mathematics. SpringerVerlag, Berlin (2005). ISBN 3-540-22617-6. MR2108619.

Lindqvist, B. H. Association of probability measures on partially ordered spaces. J. Multivariate Anal., 26 (2), 111-132 (1988). MR963827.

Lindvall, T. On Strassen's theorem on stochastic domination. Electron. Comm. Probab., 4, 51-59 (1999). MR1711599.

Lyons, R. Determinantal probability: basic properties and conjectures. In Proceedings of the International Congress of Mathematicians-Seoul 2014. Vol. IV, pp. 137-161. Kyung Moon Sa, Seoul (2014). MR3727606.

Müller, A. and Stoyan, D. Comparison methods for stochastic models and risks. Wiley Series in Probability and Statistics. John Wiley \& Sons, Ltd., Chichester (2002). ISBN 0-471-49446-1. MR1889865.

Noebels, R. A note on stochastic order of probability measures and an application to Markov processes. Z. Oper. Res. Ser. A-B, 25 (1), A35-A43 (1981). MR627158.

Pemantle, R. Towards a theory of negative dependence. Probabilistic techniques in equilibrium and nonequilibrium statistical physics. J. Math. Phys., 41 (3), 1371-1390 (2000). MR1757964.

Peres, Y., Singh, M., and Vishnoi, N. K. Random walks in polytopes and negative dependence. In 8th Innovations in Theoretical Computer Science Conference, volume 67 of LIPIcs. Leibniz Int. Proc. Inform., pp. Art. No. 50, 10. Schloss Dagstuhl. Leibniz-Zent. Inform., Wadern (2017). MR3754974.

Pitt, L. D. Positively correlated normal variables are associated. Ann. Probab., 10 (2), 496-499 (1982). MR665603.

Poinas, A., Delyon, B., and Lavancier, F. Mixing properties and central limit theorem for associated point processes. Bernoulli, 25 (3), 1724-1754 (2019). MR3961228.

Resnick, S. I. Association and multivariate extreme value distributions. Austral. J. Stat., 30 (1), 261-271 (1988). DOI: 10.1111/j.1467-842X.1988.tb00481.x.

Rolski, T. and Szekli, R. Stochastic ordering and thinning of point processes. Stochastic Process. Appl., 37 (2), 299-312 (1991). MR1102876.

Roy, R. The Russo-Seymour-Welsh theorem and the equality of critical densities and the "dual" critical densities for continuum percolation on $\mathbf{R}^{2}$. Ann. Probab., 18 (4), 1563-1575 (1990). MR1071809.

Rüschendorf, L. Stochastically ordered distributions and monotonicity of the OCfunction of sequential probability ratio tests. Math. Operationsforsch. Statist. 
Ser. Statist., 12 (3), 327-338 (1981). MR640553.

Samorodnitsky, G. Association of infinitely divisible random vectors. Stochastic Process. Appl., 55 (1), 45-55 (1995). MR1312147.

Strassen, V. The existence of probability measures with given marginals. Ann. Math. Statist., 36, 423-439 (1965). MR177430.

Szekli, R. Stochastic ordering and dependence in applied probability, volume 97 of Lecture Notes in Statistics. Springer-Verlag, New York (1995). ISBN 0-38794450-8. MR1329324.

van den Berg, J. and Jonasson, J. A BK inequality for randomly drawn subsets of fixed size. Probab. Theory Related Fields, 154 (3-4), 835-844 (2012). MR3000563. 